\title{
Using the Matrix Summability Method to Approximate the Lip $(\xi(t), p)$ Class Functions
}

\author{
Binod Prasad Dhakal ${ }^{*}$ \\ Central Department of Education (Mathematics), Tribhuvan University, Nepal \\ *Corresponding author: binod_dhakal2004@yahoo.com
}

Received October 06, 2014; Revised November 15, 2014; Accepted November 23, 2014

\begin{abstract}
Most of the summability methods are derived from the matrix means. In this paper, author has been determined the degree of approximation of certain trigonometric functions belonging to the $\operatorname{Lip}(\xi(\mathrm{t})$, p) class by matrix method.
\end{abstract}

Keywords: matrix means, degree of approximation, generalized Lipschitz class functions

Cite This Article: Binod Prasad Dhakal, "Using the Matrix Summability Method to Approximate the Lip $(\xi(\mathrm{t}), \mathrm{p})$ Class Functions." Turkish Journal of Analysis and Number Theory, vol. 2, no. 6 (2014): 198-201. doi: 10.12691/tjant-2-6-2.

\section{Introduction}

The Fourier series associate with $\mathrm{f}$ at point $\mathrm{x}$ of $2 \pi$ periodic function in $(-\pi, \pi)$ is given by

$$
f(x) \sim \frac{1}{2} a_{0}+\sum_{n=1}^{\infty}\left(\mathrm{a}_{\mathrm{n}} \cos \mathrm{nx}+\mathrm{b}_{\mathrm{n}} \sin \mathrm{nx}\right) .
$$

A function $\mathrm{f} \in \operatorname{Lip} \alpha$ if

$$
\begin{aligned}
& f(x+t)-f(x)=O\left(|t|^{\alpha}\right), \text { for } 0<\alpha \leq 1 \\
& f \in \operatorname{Lip}(\alpha, p), \text { for } 0 \leq x \leq 2 \pi, \text { if } \\
& \left(\int_{0}^{2 \pi}|f(x+t)-f(x)|^{p} d x\right)^{\frac{1}{p}}=O\left(|t|^{\alpha}\right), \text { for } 0<\alpha \leq 1 \\
& \text { and an integer } p \geq 1 .
\end{aligned}
$$

$$
\begin{gathered}
f \in \operatorname{Lip}(\xi(t), p) \text { if } \\
\left(\int_{a}^{b}|f(x+t)-f(x)|^{p} d x\right)^{\frac{1}{p}}=O(\xi(t))
\end{gathered}
$$

provided $\xi(t)$ is positive increasing function.

If $\xi(t)=t^{\alpha}$ then $\operatorname{Lip}(\xi(t), p)$ coincide with $\operatorname{Lip}(\alpha, p)$ and if $\mathrm{p} \rightarrow \infty$ in $\operatorname{Lip}(\alpha, p)$ than $\operatorname{Lip}(\alpha, p)$ reduce to $\operatorname{Lip} \alpha$.

We observed that Lip $\alpha \subseteq \operatorname{Lip}(\alpha, \mathrm{p}) \subseteq \operatorname{Lip}(\xi(\mathrm{t}), \mathrm{p})$ for $0<\alpha \leq 1$.

We define norm $\|\cdot\|_{p}$ by

$$
\|f\|_{p}=\left\{\int_{0}^{2 \pi}|f(x)|^{p} d x\right\}^{\frac{1}{p}}, p \geq 1 .
$$

The degree of approximation $\mathrm{E}_{\mathrm{n}}(\mathrm{f})$ is given by

$$
E_{n}(f)=\min \left\|t_{n}-f\right\|_{p}
$$

where $t_{n}(x)$ is a trigonometric polynomial of degree $n$.

Let $\mathrm{T}=\left(\mathrm{a}_{\mathrm{n}, \mathrm{k}}\right)$ be an infinite lower triangular matrix satisfying the condition(see, [4]) of regularity. Let $\sum_{m=0}^{\infty} u_{n}$ be an infinite series such that whose $n^{\text {th }}$ partial sum $\mathrm{s}_{n}=\sum_{k=0}^{n} u_{k}$.

The sequence-to-sequence transformation $t_{n}=\sum_{k=0}^{n} a_{n, k} s_{k}$ defines the sequence $\left\{t_{n}\right\}$ of lower triangular matrix means of the sequence $\left\{\mathrm{s}_{\mathrm{n}}\right\}$ generated by the sequence of coefficients $\left(a_{n, k}\right)$.

The series $\sum_{n=0}^{\infty} u_{n}$ is said to be summable to the sum $\mathrm{s}$ by lower triangular matrix method (see, [1]) if $\lim _{n \rightarrow \infty} t_{n}=s$.

In this paper, we use following notations.

$$
\begin{gathered}
\varphi(t)=f(x+t)+f(x-t)-2 f(x) \\
A_{n, \tau}=\sum_{k=n-\tau}^{n} a_{n, k},
\end{gathered}
$$

where $\tau=\left[\frac{1}{t}\right]$ is the greatest integer not greater than $(1 / \mathrm{t})$ and

$$
\begin{aligned}
& \mathrm{M}_{\mathrm{n}}(\mathrm{t}) \\
& =\frac{1}{2 \pi} \sum_{k=0}^{n} a_{n, k} \frac{\sin (k+1 / 2) t}{\sin (t / 2)}
\end{aligned}
$$




\section{Main Theorem}

Chandra[5] proved a theorem on the approximation of function belonging to $\operatorname{Lip}(\alpha, p)$ class by Nörlund and Riesz means. Mittal et. al. [3] extended the result of Chandra [5] by using the matrix means on same $\operatorname{Lip}(\alpha, p)$ class function. In [6], Lal \& Dhakal proved a theorem on approximation of a Lip $\alpha$ class function by matrix means.

Aim of this paper is to extend the theorems of Chandra [5], Mittal et.al. [3] and Lal \& Dhakal [6] by using matrix means on $\operatorname{Lip}(\xi(t), p)$ class functions as following way:

Theorem. Let $\mathrm{T}=\left(\mathrm{a}_{\mathrm{n} . \mathrm{k}}\right)$ be an infinite regular lower triangular matrix such that the element $\left(a_{n . k}\right)$ be nonnegative, non-decreasing with $k \leq n$. If a function $f: \mathfrak{R} \rightarrow \mathfrak{R}$ is $2 \pi$-periodic, Lebesgue integrable on $[-\pi, \pi]$ and belonging to $\operatorname{Lip}(\xi(t), p)$ class then the degree of approximation of $\mathrm{f}$ by lower triangular matrix means $t_{n}(x)=\sum_{k=0}^{n} a_{n, k} s_{k}(x)$ of its Fourier series (1.1) satisfies, for $\mathrm{n}=0,1,2,3 \ldots$,

$$
\left\|t_{n}-f\right\|_{p}=O\left((n+1)^{\frac{1}{p}} \xi\left(\frac{1}{n+1}\right)\right)
$$

provided $\xi(\mathrm{t})$ satisfies the following conditions:

$$
\begin{gathered}
{\left[\int_{0}^{\frac{1}{n+1}}\left(\frac{t|\varphi(t)|}{\xi(t)}\right)^{p} d t\right]^{\frac{1}{p}}=O\left(\frac{1}{n+1}\right),} \\
{\left[\int_{\frac{1}{n+1}}^{\pi}\left(\frac{t^{-\delta}|\varphi(t)|}{\xi(t)}\right)^{p} d t\right]^{\frac{1}{p}}=O((n+1) \delta)}
\end{gathered}
$$

where $\delta$ is an arbitrary number such that $\mathrm{q}(1-\delta)-1>0$, $\mathrm{q}$ the conjugate index of $\mathrm{p}$ and conditions (2.2) \& (2.3) hold uniformly in $\mathrm{x}$.

\section{Lemmas}

For the proof of the theorem following lemmas are required.

Lemma 1. $\mathrm{M}_{\mathrm{n}}(\mathrm{t})=\mathrm{O}(\mathrm{n}+1)$, if $0 \leq t \leq 1 /(n+1)$.

Proof. For $0 \leq t \leq 1 /(n+1), \sin (n+1) t \leq(n+1) t$,

$$
\begin{aligned}
\left|\mathrm{M}_{\mathrm{n}}(\mathrm{t})\right| & \leq\left|\frac{1}{2} \sum_{k=0}^{n} a_{n, k} \frac{(2 k+1)(t / 2)}{t}\right| \\
& \leq \frac{(2 n+1)}{4} \sum_{k=0}^{n} a_{n, k} \\
& =\mathrm{O}(\mathrm{n}+1) .
\end{aligned}
$$

Lemma 2. If $\left(a_{n, k}\right)$ is non- negative and non- decreasing with $\mathrm{k} \leq \mathrm{n}$, then, $\left|\sum_{k=0}^{n} a_{n, k} e^{i k t}\right|=O\left(A_{n, \tau}\right)$, uniformly in $0<t \leq \pi$.
Proof: Let $\tau=\left[\frac{1}{t}\right] \leq n$. Then

$$
\begin{aligned}
\left|\sum_{k=0}^{n} a_{n, k} e^{i k t}\right| & =\left|\sum_{k=0}^{n-\tau-1} a_{n, k} e^{i k t}+\sum_{k=n-\tau}^{n} a_{n, k} e^{i k t}\right| \\
& \leq\left|\sum_{k=0}^{n-\tau-1} a_{n, k} e^{i k t}\right|+\left|\sum_{k=n-\tau}^{n} a_{n, k} e^{i k t}\right|,
\end{aligned}
$$

by Abel's lemma,

$$
\begin{aligned}
\left|\sum_{k=0}^{n-\tau-1} a_{n, k} e^{i k t}\right| & \leq 2 a_{n, n-\tau-1} \max _{1 \leq k \leq n \leq n-\tau-1}\left|\frac{1-e^{i(k+1) t}}{1-e^{i t}}\right| \\
& \leq 4 a_{n, n-\tau-1}\left|\frac{e^{i t / 2}}{e^{-i t / 2}-e^{i t / 2}}\right| \\
& \leq 2 a_{n, n-\tau-1}\left(\frac{1}{\sin \frac{t}{2}}\right) \\
& \leq \frac{2 \pi a_{n, n-\tau-1}}{t}
\end{aligned}
$$

and

$$
\begin{aligned}
A_{n, \tau} & =\sum_{k=n-\tau}^{n} a_{n, k}=a_{n, n-\tau}+a_{n, n-\tau++1}+\ldots+a_{n, n} \\
& \geq a_{n, n-\tau-1}+a_{n, n-\tau-1}+\ldots+a_{n, n-\tau-1} \\
& =(\tau+1) a_{n, n-\tau-1} \\
& \geq \frac{a_{n, n-\tau-1}}{t}
\end{aligned}
$$

therefore

$$
\left|\sum_{k=0}^{n-\tau-1} a_{n, k} e^{i k t}\right| \leq 2 \pi A_{n, \tau}
$$

Also, $\left|\sum_{k=n-\tau}^{n} a_{n, k} e^{i k t}\right| \leq \sum_{k=n-\tau}^{n} a_{n, k}=A_{n, \tau}$.

Thus $\left|\sum_{k=0}^{n} a_{n, k} e^{i k t}\right| \leq(2 \pi+1) A_{n, \tau}=O\left(A_{n, \tau}\right)$.

Lemma 3. $M_{n}(t)=O\left(\frac{A_{n, \tau}}{t}\right)$, if $\frac{1}{n+1}<t \leq \pi$.

Proof: For $\frac{1}{n+1}<t \leq \pi, \sin (\mathrm{t} / 2) \geq(t / \pi)$, we have

$$
\begin{aligned}
\left|\mathrm{M}_{\mathrm{n}}(\mathrm{t})\right| & \leq\left|\frac{1}{2 t} \operatorname{Im} \sum_{k=0}^{n} a_{n, k} e^{i\left(k+\frac{1}{2}\right) t}\right| \\
& \leq \frac{1}{2 t}\left|\sum_{k=0}^{n} a_{n, k} e^{i k t}\right|\left|e^{i t / 2}\right| \\
& =O\left(\frac{A_{n, \tau}}{t}\right), \text { using lemma } 2 .
\end{aligned}
$$

\section{Proof of the Theorem}

Following [2], we have 


$$
s_{k}(x)-f(x)=\frac{1}{2 \pi} \int_{0}^{\pi} \varphi(t) \frac{\sin (k+1 / 2) t}{\sin (t / 2)} d t
$$

then,

$$
\begin{aligned}
& \sum_{k=0}^{n} a_{n, k}\left(s_{n, k}(x)-f(x)\right) \\
& =\frac{1}{2 \pi} \int_{0}^{\pi} \varphi(t) \sum_{k=0}^{n} a_{n, k} \frac{\sin (k+1 / 2) t}{\sin (t / 2)} d t
\end{aligned}
$$

or

$$
\begin{aligned}
& t_{n}(x)-f(x)=\int_{0}^{\pi} \varphi(t) M_{n}(t) d t \\
& =\left[\int_{0}^{1 /(n+1)} \varphi(t) M_{n}(t) d t+\int_{1 /(n+1)}^{\pi} \varphi(t) M_{n}(t) d t\right] \\
& =I_{1}+I_{2}, \text { say. }
\end{aligned}
$$

For, $\mathrm{I}_{1}$,

Applying Holder's inequality and fact that $\varphi(t) \in \operatorname{Lip}(\xi(t), p)$, we have

$$
\begin{aligned}
& \left|I_{1}\right| \leq\left[\int_{0}^{\frac{1}{n+1}}\left(\frac{t|\varphi(t)|}{\xi(t)}\right)^{p} d t\right]^{\frac{1}{p}}\left[\int_{0}^{\frac{1}{n+1}}\left\{\frac{\xi(t)}{t}\left(M_{n}\right)(t)\right\}^{q} d t\right]^{\frac{1}{q}} \\
& =O\left(\frac{1}{n+1}\right) O\left[\int_{0}^{\frac{1}{n+1}}\left(\frac{\xi(t)}{t}(n+1)\right)^{q} d t\right]^{\frac{1}{q}},
\end{aligned}
$$

by condition (2.2) \& Lemma 1.

Applying Second Mean Value Theorem for Integrals, we have

$$
\begin{aligned}
& =O\left(\xi\left(\frac{1}{n+1}\right)\right)\left[\int_{\epsilon}^{\frac{1}{n+1}} t^{-q} d t\right]^{\frac{1}{q}}, \\
& \text { for some } 0<\in<\frac{1}{n+1} \\
& \left.\left.=O\left(\xi\left(\frac{1}{n+1}\right)\right)\left[\left\{\frac{t^{-q+1}}{-q+1}\right\}^{\frac{1}{n+1}}\right]^{\frac{1}{q}}\right]^{\frac{1}{q}} \xi\left(\frac{1}{n+1}\right)\right) \\
& =O\left((n+1)^{\frac{1}{p}} \xi\left(\frac{1}{n+1}\right)\right) \\
& =O\left((n+1)^{\frac{1}{p}}\right. \\
& \because \frac{1}{p}+\frac{1}{q}=1 .
\end{aligned}
$$

Applying Holder's inequality, condition (2.3) and Lemma 2.

$$
\begin{aligned}
& \left|I_{2}\right| \leq\left[\int_{\frac{1}{n+1}}^{\pi}\left(\frac{t^{-\delta}|\varphi(t)|}{\xi(t)}\right)^{p} d t\right]^{\frac{1}{p}}\left[\int_{\frac{1}{n+1}}^{\pi}\left(\frac{\xi(t)}{t^{-\delta}}\left(M_{n}\right)(t)\right)^{q} d t\right]^{\frac{1}{q}} \\
& =O\left((n+1)^{\delta}\right) O\left[\left[\int_{\frac{1}{n+1}}^{\pi}\left(\frac{\xi(t)}{t^{-\delta}} \frac{A_{n, \tau}}{t}\right)^{q} d t\right]^{\frac{1}{q}}\right] \\
& =O\left((n+1)^{\delta}\right)\left[\int_{\frac{1}{\pi}}^{n+1}\left(\frac{\xi\left(\frac{1}{u}\right) A_{n, u}}{\left(\frac{1}{u}\right)^{(1-\delta)}}\right)^{q} \frac{d u}{u^{2}}\right]^{\frac{1}{q}},
\end{aligned}
$$

taking $t=\frac{1}{u}$

$$
\begin{aligned}
& =O\left((n+1)^{\delta} \xi\left(\frac{1}{n+1}\right) A_{n, n}\right)\left[\int_{\frac{1}{\pi}}^{n+1} u^{q(1-\delta)-2} d u\right]^{\frac{1}{q}} \\
& =O\left((n+1)^{\delta} \xi\left(\frac{1}{n+1}\right)\right)\left[\left(\frac{u^{q(1-\delta)-1}}{q(1-\delta)-1}\right)_{\frac{1}{\pi}}^{n+1}\right]^{\frac{1}{q}} \\
& =O\left((n+1)^{\delta} \xi\left(\frac{1}{n+1}\right)\right)\left((n+1)\left(\frac{1-\frac{1}{q}}{)^{1}}\right)^{1-\frac{1}{q}} \xi\left(\frac{1}{n+1}\right)\right)=O\left((n+1)^{\frac{1}{p}} \xi\left(\frac{1}{n+1}\right)\right) \\
& =O\left((n+1)^{(}{ }^{\frac{1}{n}}\right)
\end{aligned}
$$

Combining the conditions $(4.1)-(4.3)$, we have

$$
\left|t_{n}-f(x)\right|=O\left((n+1)^{\frac{1}{p}} \xi\left(\frac{1}{n+1}\right)\right) .
$$

Now,

$$
\begin{aligned}
& \left\|t_{n}-f\right\|_{p}=\left[\int_{0}^{2 \pi}|t(x)-f(x)|^{p} d x\right]^{\frac{1}{p}} \\
& =O\left[\int_{0}^{2 \pi}\left\{(n+1)^{\frac{1}{p}} \xi\left(\frac{1}{n+1}\right)\right\}^{p} d x\right]^{\frac{1}{p}} \\
& =O\left((n+1)^{\frac{1}{p}} \xi\left(\frac{1}{n+1}\right)\right)\left[\int_{o}^{2 \pi} d x\right]^{\frac{1}{p}} \\
& =O\left((n+1)^{\frac{1}{p}} \xi\left(\frac{1}{n+1}\right)\right)
\end{aligned}
$$

This completes the proof of the theorem. 


\section{Corollaries}

Following corollaries can be derived from the theorem. Corollary 1. If $\xi(\mathrm{t})=\mathrm{t}^{\alpha}$ then degree of approximation of a function $f \in \operatorname{Lip}(\alpha, p)$ class is given by

$$
\left\|t_{n}-f\right\|_{p}=O\left(\frac{1}{(n+1)^{\alpha-\frac{1}{p}}}\right)
$$

for $1 \leq p<\infty, \frac{1}{p}<\alpha \leq 1$

Corollary 2. If $\xi(\mathrm{t})=\mathrm{t}^{\alpha}$ with $\mathrm{p} \rightarrow \infty$, then degree of approximation of a function $f \in \operatorname{Lip} \alpha$ class is given by

$$
\left\|t_{n}-f\right\|_{\infty}=O\left(\frac{1}{(n+1)^{\alpha}}\right) \text {, for } 0<\alpha<1 .
$$

\section{References}

[1] A. Zygmund, Trigonometric series, Cambridge University Press, 1959.

[2] E. C. Titchmarsh, Theory of functions, Oxford University Press, 1939.

[3] M. L Mittal, B. E. Rhoades, V. N. Mishra and U. Shing, Using infinite matrices to functions of class Lip $(\alpha, p)$ using trigonometric polynomials, J. Math. Anal. Appl, 326(2007), 667-676.

[4] O.Töeplitz, Über allgemeine lineare Mittelbildungen, Prace mat. fiz., 22(1913), 113-119.

[5] P. Chanrda, Trigonometric approximation of function in $L_{p}$-norm, J. Math. Anal. Appl, 275(2002), 13-676.

[6] S. Lal and B. P. Dhakal, On Approximation of functions belonging to Lipschitz class by triangular matrix method of Fourier series, Int. Journal of Math. Analysis, 4(21), 2010, 1041-1047. 